\title{
Comparison between lab variability and in silico prediction errors for the unbound fraction of drugs in human plasma
}

\section{Urban Fagerholm, Ola Spjuth \& Sven Hellberg}

To cite this article: Urban Fagerholm, Ola Spjuth \& Sven Hellberg (2021) Comparison between lab variability and in silico prediction errors for the unbound fraction of drugs in human plasma, Xenobiotica, 51:10, 1095-1100, DOI: 10.1080/00498254.2021.1964044

To link to this article: https://doi.org/10.1080/00498254.2021.1964044

曲 Published online: 13 Aug 2021.

Submit your article to this journal 준

LIII Article views: 90

Q View related articles $\longleftarrow$

View Crossmark data \ulcorner

Citing articles: 1 View citing articles $₫$ 


\title{
Comparison between lab variability and in silico prediction errors for the unbound fraction of drugs in human plasma
}

\author{
Urban Fagerholm $^{\mathrm{a}}$ (D), Ola Spjuth ${ }^{\mathrm{a}, \mathrm{b}}$ (D) and Sven Hellberg ${ }^{\mathrm{a}}$

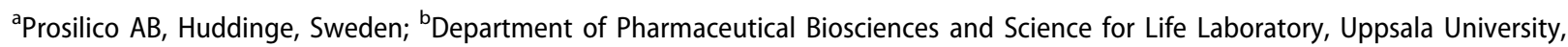 \\ Uppsala, Sweden
}

\section{ABSTRACT}

1. Variability of the unbound fraction in plasma $\left(f_{u}\right)$ between labs, methods and conditions is known to exist. Variability and uncertainty of this parameter influence predictions of the overall pharmacokinetics of drug candidates and might jeopardise safety in early clinical trials. Objectives of this study were to evaluate the variability of human in vitro $f_{u}$-estimates between labs for a range of different drugs, and to develop and validate an in silico $\mathrm{f}_{\mathrm{u}}$-prediction method and compare the results to the lab variability.

2. A new in silico method with prediction accuracy $\left(Q^{2}\right)$ of 0.69 for $\log f_{u}$ was developed. The median and maximum prediction errors were 1.9- and 92-fold, respectively. Corresponding estimates for lab variability (ratio between $\max$ and $\min f_{u}$ for each compound) were 2.0- and 185fold, respectively. Greater than 10-fold lab variability was found for 14 of 117 selected compounds.

3. Comparisons demonstrate that in silico predictions were about as reliable as lab estimates when these have been generated during different conditions. Results propose that the new validated in silico prediction method is valuable not only for predictions at the drug design stage, but also for reducing uncertainties of $\mathrm{f}_{\mathrm{u}}$-estimations and improving safety of drug candidates entering the clinical phase.
ARTICLE HISTORY

Received 8 July 2021

Revised 30 July 2021

Accepted 31 July 2021

\section{KEYWORDS}

Plasma protein binding; unbound fraction in plasma; variability; in silico;

QSAR; prediction

\section{Introduction}

The degree of plasma protein binding and its counterpart, the unbound fraction in plasma $\left(f_{\mathrm{u}}\right)$, are important determinants for the pharmacokinetics (PK), exposure profiles, pharmacodynamics (PD), safety and doses of drugs. $F_{u}$ is usually estimated in in vitro experiments, and the higher consistency between such estimates and the in vivo situation, the better PK, exposures, PD, safety and doses of candidate drugs in early clinical studies will be predicted. Poorly estimated and uncertain $f_{\mathrm{u}}$-estimates jeopardise safety in early clinical studies, and could potentially be the main reason for failure to reach an adequate exposure profile of a new drug candidate. This is of concern mainly for compounds that bind strongly to plasma proteins (accurate $\mathrm{f}_{\mathrm{u}}$-determination is a challenge mainly for highly bound compounds), bind to the equipment used for measurements, have solubility limitations and/or demonstrate concentration-dependencies for binding in the clinically relevant concentration range.

Petersson et al. (Sohlenius-Sternbeck et al. 2010; Yamagata et al. 2017) have shown that cherry-picking of favourable $f_{\mathrm{u}}$-values can have a significant impact for the correlation between predicted and observed hepatic clearance $\left(\mathrm{CL}_{H}\right)$ of drugs. In two separate studies, they used different $\mathrm{f}_{\mathrm{u}}$-estimates for $\mathrm{CL}_{\mathrm{H}}$-predictions of 16 compounds
(2.5- and 10-fold average and max differences, respectively), and the selection for the second study had a dramatic positive effect on the correlation coefficient and the apparent predictive power. Thus, the uncertainty of $f_{u}$-estimates not only influences the actual predictions, but also introduces a potential for retrospective selection bias.

Several methods for determination of $f_{u}$ exist, including equilibrium dialysis, ultrafiltration, ultracentrifugation, turbulent flow chromatography, biosensors, fluorescence spectroscopy, capillary electrophoresis, high-performance liquid chromatography using columns containing immobilised plasma proteins and microdialysis (Cohen 2004). These have their specific advantages and disadvantages. Ultrafiltration and equilibrium dialysis are the most commonly used methods today. Ultrafiltration, which allows automation and rapid determinations, is highly dependent on the extent of nonspecific binding of compounds to plastics and the ultrafiltration membrane surface (Kwon 2002; Cohen 2004). The more time consuming equilibrium dialysis is generally accepted as a more accurate method, especially for highly bound compounds (Kwon 2002). However, non-specific binding of compounds to dialysis devices and membranes is also a potential problem with this technique (Kwon 2002). According to Kariv et al. (2001), it is recommended that the equilibrium dialysis assay is not used for compounds with a solubility of less 


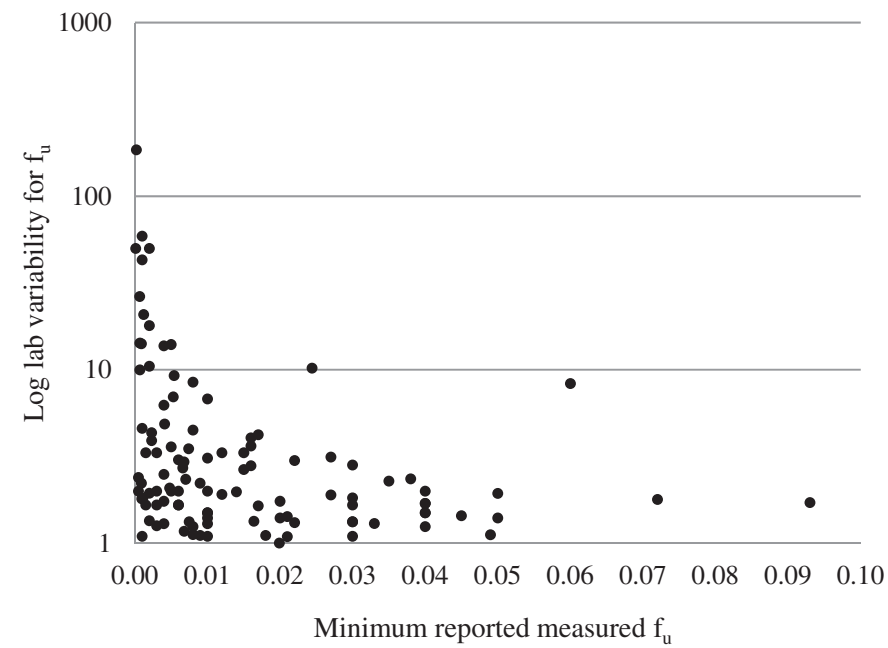

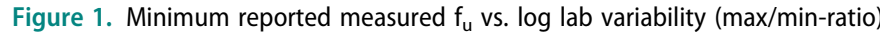
for $f_{u}$ of compounds with high degree of plasma protein binding $\left(f_{u}<0.10\right)$.

than $5 \mu \mathrm{M}$ at $37^{\circ} \mathrm{C}$ as the insoluble compound will not be able to freely cross the membrane. Another limitation with this technique is the increase of plasma protein concentration in the plasma sample as plasma water is filtrated (Zeitlinger et al. 2011).

An alternative to $f_{u}$-measurements at labs is in silico predictions. One successful example is that by Zhivkova and Doytchinova (2012). They created an in silico model where $57 \%$ of predictions of $f_{u}$ for a set of acids had an error of maximally 2-fold and a predictive accuracy $\left(\mathrm{Q}^{2}\right)$ of 0.74 . Another example is an in silico model developed by Watanabe et al. (2018) which reached $Q^{2}$-estimates of 0.67 to 0.72 for different test sets.

The first objective of this study was to evaluate the variability of human in vitro $\mathrm{f}_{\mathrm{u}}$-estimates between labs, methods and conditions for a range of different drugs. The second objective was to develop and validate an in silico method for prediction of $\mathrm{f}_{\mathrm{u}}$ in human plasma and compare the results to the variability shown for lab data.

\section{Materials and methods}

\section{Collection of in vitro $f_{u}$-data}

The research literature was searched for drugs with $f_{\mathrm{u}}$-estimates obtained in at least two different studies. The aim was to find at least 100 compounds with low, moderate and high $f_{u}$, with particular focus on collecting data for highly bound compounds. Multiple in vitro $f_{u}$-data were found for 117 compounds, and the minimum reported $\mathrm{f}_{\mathrm{u}}$ for these ranged from 0.00010 to 0.72 (Hervé et al. 1994; Iwatsubo et al. 1997; Obach 1999; Goodman Gilman 2001; Kratochwil et al. 2002; Shibata et al. 2002. Riley et al. 2005; Brown et al. 2007; De Buck et al. 2007; McGinnity et al. 2007; Abe et al. 2008; Obach et al. 2008; Hallifax et al. 2010; Sohlenius-Sternbeck et al. 2010; Uchimura et al. 2010; Poulin et al. 2011; Jones et al. 2012; Zhivkova and Doytchinova 2012; Schlicht and Miller 2013; Yamagata et al. 2017).

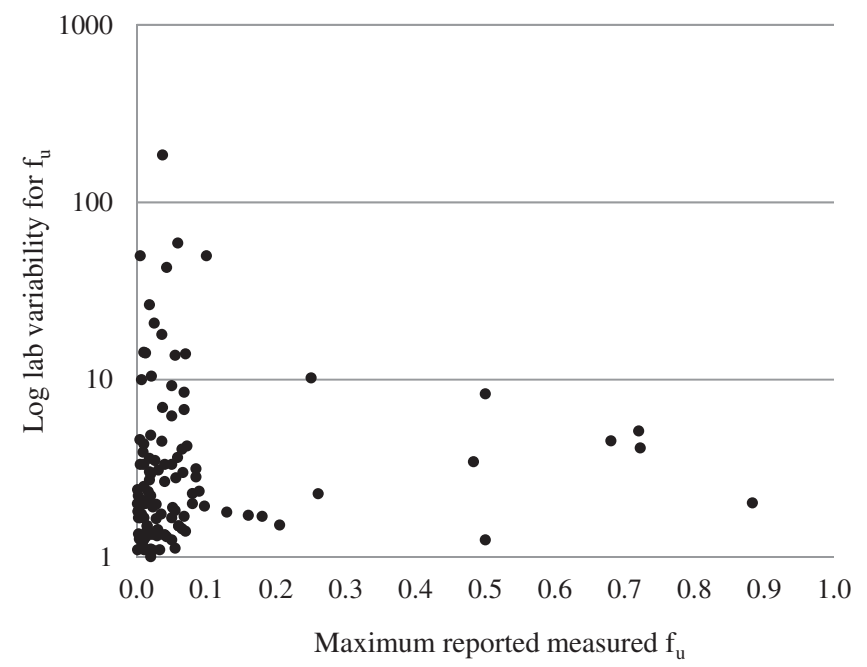

Figure 2. Maximum reported measured $f_{u} v s$, log lab variability (max/min-ratio) for $f_{u}$ of the 117 selected compounds.

\section{Determination of lab variability}

The ratios between the highest and lowest reported $\mathrm{f}_{\mathrm{u}}$-estimates for each compound were used as a measurement of lab variability. No account was taken for methodologies and concentrations used. An alternative used to determine lab variability for each compound was to establish ratios between maximum and mean values and mean and minimum values, and selecting the greatest of these two.

\section{Development of an in silico method for prediction of $f_{u}$}

Based on collected $\mathrm{f}_{\mathrm{u}}$-estimates for compounds with a maximum molecular weight (MW) of $750 \mathrm{~g} / \mathrm{mole}$ from the literature (the lowest of reported values for each compound were selected; lowest values were chosen as these were also used for assessing lab variability and create a larger span) and quantitative structure-activity relationship/partial least squares (QSAR/PLS) modelling, a regression-like in silico model for $\log f_{\mathrm{u}}$-predictions was developed (SIMCA version 16 2019). The predictive power of the resulting model was validated by a 10 -fold cross validation procedure, which we normally use in our work, assures that each compound has a forward-looking prediction, and also appears to be common/ default in this field.

\section{Comparison between lab variability and in silico prediction errors}

Lab variability and in silico prediction errors were compared both as a whole (selected in vitro data for compounds with different reported estimates vs. results for all compounds used for and predicted with the in silico model) and head-tohead (for compounds with the greatest lab variability and/or in silico prediction errors). Results are demonstrated as correlations and ratios (between maximum and minimum $\log f_{u}$ for in vitro data, and predicted and observed $\log f_{u}$ for in silico results). 


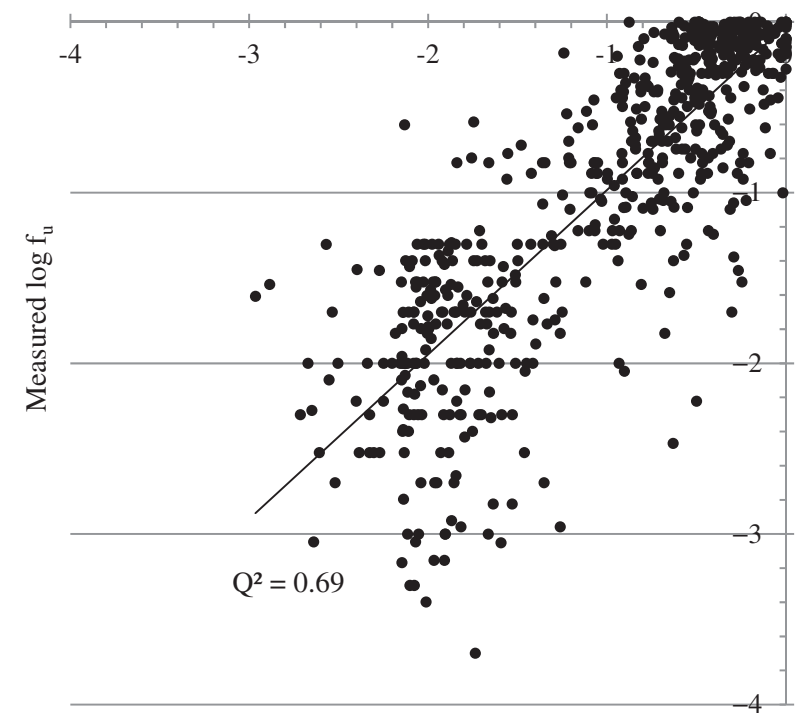

Predicted $\log \mathrm{f}_{\mathrm{u}}$

Figure 3. Predicted (in silico) vs. measured . $\log \mathrm{f}_{\mathrm{u}}(n=599)$.

\section{Results}

\section{Variability of in vitro $f_{u}$}

Figures 1 and 2 demonstrate the relationships between minimum and maximum measured $f_{u}$ vs. log maximum/minimum $\mathrm{f}_{\mathrm{u}}$-ratios (lab variability) for the selected 117 drugs, respectively. As can be seen, there is an apparent relationship between the level of $f_{u}$ and lab variability (with greater lab variability for highly bound compounds). A 185-fold lab variability was found for the highly lipophilic and highly bound drug amiodarone (minimum and maximum reported $f_{u}$ are 0.00020 and 0.037 , respectively). Greater than 10-fold lab variability was found for 14 compounds with $f_{u}<0.1$, and 5- to 8-fold lab variability was found for a few compounds with $f_{u} 0.2$ to $0.7 ; 19 \%$ of the compounds in the selected dataset had a lab variability exceeding 5 -fold. The mean and median lab variability for this set of compounds was 6.8- and 2.0-fold, respectively.

\section{In silico method for prediction of $f_{u}$}

The final in silico model, based on human $\mathrm{f}_{\mathrm{u}}$-data for 599 drugs with a MW up to $750 \mathrm{~g} / \mathrm{mole}$ (Hervé et al. 1994; Iwatsubo et al. 1997; Obach 1999; Goodman Gilman 2001; Kratochwil et al. 2002; Shibata et al. 2002; Riley et al. 2005; Brown et al. 2007; De Buck et al. 2007; McGinnity et al. 2007; Abe et al. 2008; Obach et al. 2008; Hallifax et al. 2010; Sohlenius-Sternbeck et al. 2010; Uchimura et al. 2010; Poulin et al. 2011; Jones et al. 2012; Zhivkova and Doytchinova 2012; Schlicht and Miller 2013; Yamagata et al. 2017 (major source of $\mathrm{f}_{\mathrm{u}}$-data; $\left.n=553\right)$ ) and with 7 components/dimensions, has a $Q^{2}$-value of 0.69 (for $\log f_{u}$ i Figure 3). Mean, median and maximum ratios between predicted and lowest measured estimates are 3.4-, 1.9- and 92-fold (for amiodarone), respectively (Figure 4); 55, 74, 89 and $95 \%$ of ratios between predicted and lowest measured estimates were $<2-,<3-,<5$ - and $<10$-fold, respectively.

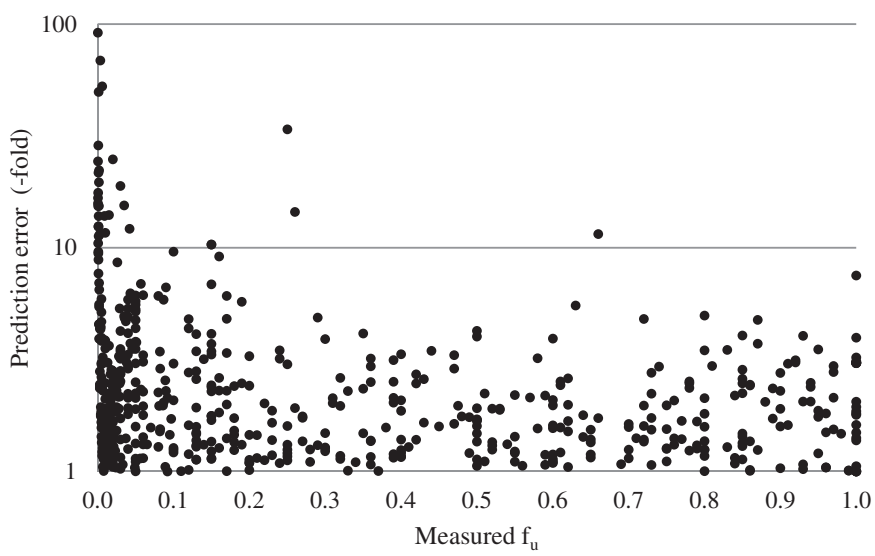

Figure 4. Measured $f_{u}$ vs. log prediction error (-fold) of $f_{u}$ for the in silico method $(n=599)$.

\section{Lab variability vs. in silico prediction error}

The in silico method demonstrated higher correlation for predicted vs. measured minimum $\log f_{u}\left(Q^{2}=0.69 ; n=599\right)$ than minimum vs. maximum measured $\log f_{u}\left(R^{2}=0.61 ; n=117\right)$. Median, mean and maximum ratios for predicted vs. measured minimum $\mathrm{f}_{\mathrm{u}}$ (1.9-, 3.4- and 92-fold, respectively) were also lower than corresponding lab variability $(2.0-, 6.8-$ and 185 -fold, respectively) (Figure 5). When assuming that the mean measured $f_{u}$ for each compound is correct, and minimum and maximum estimates demonstrate the lab variability, there was mean, median and maximum lab variability of 3.3-, 1.5- and 47-fold, respectively. For 19 highly bound compounds with lab variability and/or prediction error $>10$-fold, similar results were shown for lab variability and/or prediction error (Table 1). Depending on how results are compared (in silico prediction errors vs. max/min-, max/mean or mean/ min-ratios at labs), in silico prediction errors were greater than lab variability for 7 or 11 of these 19 compounds. The in silico method does, however, not appear to demonstrate an advantage vs. lab variability at higher $f_{u}$ (Figures 2 and 4).

\section{Discussion}

Results demonstrate that extensive differences between labs are common, especially for highly protein bound compounds - 26- to 185-fold lab variability was found for six highly bound compounds. Such differences are likely to have significant impact on predicted PK, PD, exposures and doses and for the safety in early clinical studies of drug candidates. A 5800-fold overexposure of systemic exposure has been demonstrated after the first intravenous dose of a highly bound anticancer compound, and plasma protein binding was an important factor (Fuse et al. 1998). It is recommended that such differences, uncertainties and potential errors are considered when setting the initial dose in first clinical studies, to avoid overexposures and unwanted side effects.

It must be taken into account that non-specific binding to plastics and membranes could have affected $\mathrm{f}_{\mathrm{u}}$-measurements, and played a role in significant lab differences and prediction errors. The 185 -fold lab variability and 92 -fold 


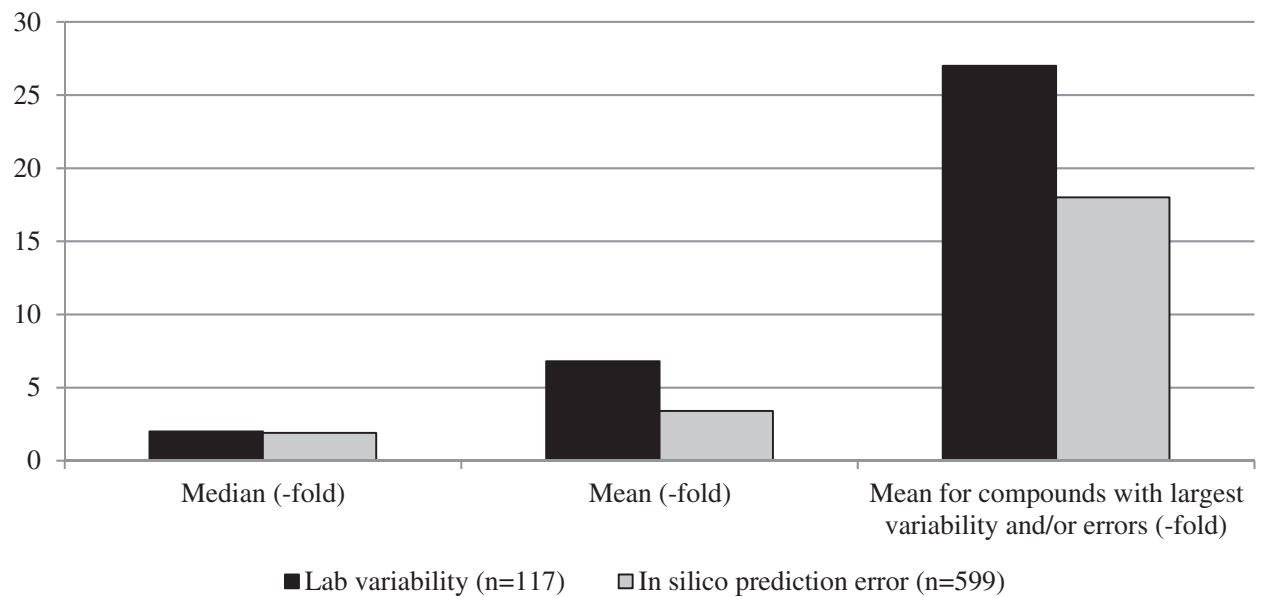

Figure 5. Median and mean (including mean for the 19 compounds with the largest lab variability and/or prediction errors) lab variability vs. in silico prediction errors.

Table 1. Comparison between lab variability and in silico prediction errors for 19 compounds with $>10$-fold lab variability and/ or prediction errors for $f_{u}$.

\begin{tabular}{|c|c|c|c|c|}
\hline & Lab variability & & In silico & Ratio \\
\hline Compound & $\operatorname{Max} / \mathrm{min}^{\mathrm{a}}$ & Max/mean or mean $/ \mathrm{min}^{\mathrm{b}}$ & Prediction error $^{c}$ & Lab vs. in silico $^{d}$ \\
\hline Amiodarone & 185 & 47 & 92 & $2.0 / 0.51$ \\
\hline Valsartan & 59 & 39 & 7.7 & $7.7 / 5.1$ \\
\hline Tamoxifen & 50 & 26 & 44 & $1.1 / 0.59$ \\
\hline Diclofenac & 50 & 8.2 & 22 & 2.3/0.37 \\
\hline Tenidap & 43 & 22 & 17 & $2.5 / 1.3$ \\
\hline Naproxen & 26 & 7.9 & 7.1 & $3.7 / 1.1$ \\
\hline Gemfibrozil & 18 & 9.8 & 7.0 & $2.6 / 1.4$ \\
\hline Oxaprozin & 14 & 5.6 & 11 & $1.3 / 0.51$ \\
\hline Diflunisal & 14 & 5.9 & 29 & $0.48 / 0.20$ \\
\hline Amlodipine & 14 & 6.7 & 1.8 & 7.8/3.7 \\
\hline Indomethacin & 14 & 4.7 & 1.9 & $7.4 / 2.5$ \\
\hline Glyburide & 11 & 5.8 & 1.5 & $7.3 / 3.9$ \\
\hline Tenidap & 10 & 3.5 & 18 & $0.56 / 0.19$ \\
\hline Calcitrol & 4.6 & 2.8 & 13 & $0.35 / 0.19$ \\
\hline Bepridil & 3.3 & 2.1 & 15 & $0.22 / 0.14$ \\
\hline Tolcapone & 2.4 & 1.9 & 17 & $0.14 / 0.11$ \\
\hline Atovaquone & 2.0 & 1.8 & 16 & $0.13 / 0.11$ \\
\hline Bromfenac & 1.8 & 1.4 & 14 & $0.13 / 0.10$ \\
\hline Quercetin & 1.1 & 1.1 & 14 & $0.08 / 0.08$ \\
\hline Mean & 27 & 11 & 18 & - \\
\hline Median & 14 & 5.8 & 14 & - \\
\hline
\end{tabular}

${ }^{a}$ Ratios between maximum and minimum measured reported estimates.

${ }^{b}$ Ratios between maximum and mean or mean and minimum measured reported estimates (highest of the two are shown).

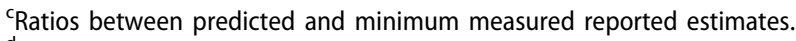

${ }^{\mathrm{d}}$ Ratios between lab variability (left estimate $=$ vs. $\mathrm{max} / \mathrm{min}$; right estimate $=$ vs. highest of $\mathrm{max} / \mathrm{mean}$ or $\mathrm{mean} / \mathrm{min}$ ) and in silico prediction error.

in silico overprediction that was found for the highly lipophilic and highly bound drug amiodarone could possibly be such an example.

One way to ensure optimal $\mathrm{f}_{\mathrm{u}}$-estimation and safe dosing is to use standardised $f_{u}$-measurement methods with reference compound-corrected $\mathrm{f}_{\mathrm{u}}$-estimates.

A more extensive analysis and review of the plasma protein binding field, including comparisons between assays and experimental set-ups, would have been valuable for method selection, and for optimising predictions of PK, exposures and doses and in silico prediction methodologies.

The new in silico method, which produces $\mathrm{f}_{\mathrm{u}}$-estimates with an uncertainty similar to the estimated lab variability, could also be useful for improving predictions and reducing uncertainties, especially for highly bound compounds. Its performance (e.g. $Q^{2}=0.69$ and $55 \%$ within 2 -fold prediction error) was similar to that obtained by others. For example, Zhivkova and Doytchinova (2012) reached a $Q^{2}$ of 0.74 and $57 \%$ within 2-fold prediction error with a QSAR model for 132 acids (cross-validation; 16 descriptors in model). However, they removed six outliers from model, of which four with $\mathrm{f}_{\mathrm{u}}<0.003$ did not obey the consensus model. These include verlukast, bromfenac and telmisartan, which showed 24-, 14- and 1.8-fold prediction errors with our new method, respectively. The implication of compound removal is that their results for acids were somewhat exaggerated. Watanabe et al. (2018) reached $Q^{2}$-estimates of 0.67 to 0.72 with their in silico prediction approach based on a dataset of 2738 compounds. With their method, there were, however, significant over- and underprediction trends at lower and higher $f_{u}$, respectively. Our method produced similar prediction accuracy as these methods by others based on smaller 
and larger datasets, but without trends. Therefore, conclusions drawn from our own results are supported by others.

The significant lab variability (averaging 6.8- or 3.3-fold depending on estimation method) and moderate correlation between minimum and maximum reported $\log f_{\mathrm{u}}$-values $\left(R^{2}=0.61\right)$, demonstrated in the present study also set a limit to how well $\mathrm{CL}_{H}$ and renal clearance can be predicted.

Advantages with the new, fully validated in silico method include high productivity and cost-efficiency, and that sufficiently reliable $\mathrm{f}_{\mathrm{u}}$-data (at least as reliable as in vitro-estimates) can be produced before drug synthesis and without the use of lab work. Since this method produces one $f_{\mathrm{u}}$-estimate for each compound, selection bias for retrospective cherry-picking of favourable data is also removed.

\section{Conclusions}

Significant lab variability was demonstrated, especially for highly bound compounds. About one-fifth of selected compounds had a lab variability exceeding 5 -fold, with a maximum of 185 -fold. Such differences are likely to have significant impact on predictions of clinical PK, PD, doses and exposures and for safety in first-time-in-man studies. Results suggest that these, in particular for highly bound compounds, should be considered. The new validated in silico prediction method, with prediction errors of the same size as lab variability and accuracy similar to in silico methods developed by others, is valuable for reducing uncertainties of $f_{\mathrm{u}}$-estimations and improving safety of drug candidates entering the clinical phase.

\section{Disclosure statement}

Urban Fagerholm, Ola Spjuth and Sven Hellberg declare shares in Prosilico $A B$, a Swedish company that develops solutions for human clinical ADME/PK predictions.

\section{ORCID}

Urban Fagerholm (D) http://orcid.org/0000-0001-6300-359X

Ola Spjuth (D) http://orcid.org/0000-0002-8083-2864

\section{References}

Abe K, Bridges A, Yue W, Brouwer KL. 2008. In vitro biliary clearance of angiotensin II receptor blockers and 3-hydroxy-3-methylglutaryl-coenzyme $A$ reductase inhibitors in sandwich-cultured rat hepatocytes: comparison with in vivo biliary clearance. J Pharmacol Exp Ther. 326(3):983-990.

Brown HS, Griffin M, Houston JB. 2007. Evaluation of cryopreserved human hepatocytes as an alternative in vitro system to microsomes for the prediction of metabolic clearance. Drug Metab Dispos. 35(2): 293-301.

Cohen LH. 2004. Plasma protein-binding methods in drug discovery. In: Yan Z, Caldwell GW, editors. Optimizing drug discovery - in vitro methods. Totowa (NJ): Humana Press Inc.; p. 111-122.

De Buck SS, Sinha VK, Fenu LA, Nijsen MJ, Mackie CE, Gilissen RAHJ. 2007. Prediction of human pharmacokinetics using physiologically based modeling: a retrospective analysis of 26 clinically tested drugs. Drug Metab Dispos. 35(10):1766-1780.
Fuse $E$, Tanii $H$, Kurata $N$, Kobayashi $H$, Shimada $Y$, Tamura $T$, Sasaki $Y$, Tanigawara Y, Lush RD, Headlee D, et al. 1998. Unpredicted clinical pharmacology of UCN-01 caused by specificbinding to human alfa-1acid glycoprotein. Cancer Res. 58:3248-3253.

Goodman Gilman A, In Hardman JG, Limbird LE. 2001. Goodman Gilman. Editors Goodman and Gilman's: the pharmacological basis of therapeutics. Int. ed. New York, NY: McGraw-Hill.

Hallifax D, Foster JA, Houston JB. 2010. Prediction of human metabolic clearance from in vitro systems: retrospective analysis and prospective view. Pharm Res. 27(10):2150-2161.

Hervé F, Urien S, Albengre E, Duché JC, Tillement J-P. 1994. Drug binding in plasma. A summary of recent trends in the study of drug and hormone binding. Clin Pharmacokinet. 26(1):44-58.

Iwatsubo T, Hirota N, Ooie T, Suzuki H, Shimada N, Chiba K, Ishizaki T, Green CE, Tyson CA, Sugiyama Y. 1997. Prediction of in vivo drug metabolism in the human liver from in vitro metabolism data. Pharmacol Ther. 73(2):147-171.

Jones H, Barton H, Lai Y, Bi Y, Kimoto E, Kempshall S, Tate S, El-Kattan A, Houston JB, Galetin A, et al. 2012. Mechanistic pharmacokinetic modeling for the prediction of transporter-mediated disposition in humans from sandwich culture human hepatocyte data. Drug Metab Dispos. 40(5):1007-1017.

Kariv I, Cao H, Oldenburg KR. 2001. Development of a high throughput equilibrium dialysis method. J Pharm Sci. 90(5):580-587.

Kratochwil NA, Huber W, Müller F, Kansy M, Gerber PR. 2002. Predicting plasma protein binding of drugs: a new approach. Biochem Pharmacol. 64(9):1355-1374.

Kwon Y, 2002. Protein binding. In: Y. Kwon, editor. Handbook of essential pharmacokinetics, pharmacokinetics, and metabolism for industrial scientists. New York, Boston, Dordrecht, London and Moscow: Kluwer Academic Publishers; p. 107-111.

McGinnity DF, Collington J, Austin RP, Riley RJ. 2007. Evaluation of human pharmacokinetics, therapeutic dose and exposure predictions using marketed oral drugs. Current Drug Met. 8:463-479.

Obach RS. 1999. Prediction of human clearance of twenty-nine drugs from hepatic microsomal intrinsic clearance data: an examination of in vitro half-life approach and nonspecific binding to microsomes. Drug Metab Disp. 27:1350-1359.

Obach RS, Lombardo F, Waters NJ. 2008. Trend analysis of a database of intravenous pharmacokinetic parameters in humans for 670 drug compounds. Drug Metab Dispos. 36(7):1385-1405.

Poulin P, Jones RDO, Jones HM, Gibson CR, Rowland M, Chien JY, Ring BJ, Adkison KK, Ku MS, He H, et al. 2011. PHRMA CPCDC Initiative on predictive models of human pharmacokinetics, Part 5: prediction of plasma concentration-time profiles in human by using the physiologically-based pharmacokinetic modeling approach . J Pharm Sci. 100(10):4127-4157.

Riley RJ, McGinnity DF, Austin RP. 2005. A unified model for predicting human hepatic, metabolic clearance from in vitro intrinsic clearance data in hepatocytes and microsomes. Drug Metab Dispos. 33(9): 1304-1311.

Schlicht KE, Miller VP. 2013. Ultrafast plasma protein binding analysis using the agilent rapid fire high-throughput mass spectrometry system and accurate mass Q-TOF. Wakefield (MA): Agilent Technologies, Inc.

Shibata Y, Takahashi H, Chiba M, Ishii Y. 2002. Prediction of hepatic clearance and availability by cryopreserved human hepatocytes: an application of serum incubation method. Drug Metab Dispos. 30(8): 892-896.

SIMCA version 16. 2019. Sartorius Stedim Umetrics, Umeå.

Sohlenius-Sternbeck A-K, Afzelius L, Prusis P, Neelissen J, Hoogstraate J, Johansson J, Floby E, Bengtsson A, Gissberg O, Sternbeck J, et al. 2010. Evaluation of the human prediction of clearance from hepatocyte and microsome intrinsic clearance for 52 drug compounds. Xenobiotica. 40(9):637-649.

Uchimura T, Katob M, Saitoc T, Kinoshitac H. 2010. Prediction of human blood-to-plasma drug concentration ratio. Biopharm Drug Dispos. 31: 286-297.

Watanabe R, Esaki T, Kawashima H, Natsume-Kitatani Y, Nagao C, Ohashi R, Mizuguchi K. 2018. Predicting fraction unbound in human plasma 
from chemical structure: improved accuracy in the low value ranges. Mol Pharm. 15(11):5302-5311.

Yamagata T, Zanelli U, Gallemann D, Perrin D, Dolgos H, Petersson C. 2017. Comparison of methods for the prediction of human clearance from hepatocyte intrinsic clearance for a set of reference compounds and an external evaluation set. Xenobiotica. 47(9):741-751.
Zeitlinger MA, Derendorf $\mathrm{H}$, Mouton JW, Cars O, Craig WA, Andes D, Theuretzbacher U. 2011. Protein binding: do we ever learn? Antimicrob Agents Chemother. 55(7):3067-3074.

Zhivkova Z, Doytchinova I. 2012. Quantitative structure-plasma protein binding relationships of acidic drugs. J Pharm Sci. 101(12): 4627-4641. 